KULTURA - MEDIA - TEOLOGIA

ISSN 2081-89-71

2018 nr 32, s. 179-191

\title{
Jacek Rosa
}

Wyższa Szkoła Policji w Szczytnie

\section{Issues of early life in postmodern philosophies according to Hugo Tristram Engelhardt}

\author{
Zagadnienia dotyczące początku życia w filozofii \\ postmodernistcznej według Hugo Tristrama Engelhardta
}

\begin{abstract}
:
The contemporary view on the beginning of life requires good comprehension of who human is as well as his beliefs. The

article presents selected thoughts of Hugo Tristam Engelhardt on the problem of the beginning of life and artificial insemination. These reflections come from Engelhardt's utilitarian standpoint.

The point of view is confronted with a line of thoughts of other authors, who represent personalistic bioethics to show how to manage proposals from a strict ethical point of view.
\end{abstract}

KEY WORDS:

birth of life, artificial insemination, H.T. Engelhardt

\section{STRESZCZENIE:}

Współczesne spojrzenie na początek życia rodzi potrzebę, dobrego zrozumienia kim jest człowiek i jakie ma wartości. W powyższym artykule zostaną przedstawione wybrane myśli dotyczące spojrzenia Hugo Tristrama Engelhardta na zagadnienie początku życia i sztucznego zapłodnienia. Te refleksje pochodzą z utylitarystycznego stanowiska Engelhardta. I ten punkt spojrzenia jest skonfrontowany ze sposobem myślenia przedstawicieli bioetyki personalistycznej, aby pokazać jak radzić sobie z propozycjami z punktu widzenia ściśle etycznego.

\section{SŁOWA KLUCZOWE:}

początek życia, sztuczne zapłodnienie, H.T. Engelhardt

\section{PAROLE CHIAVE:}

inizio vita, fecondazione assistita, H.T. Engelhardt

\section{LE QUESTIONI RELATIVE ALL'INIZIO DELLA VITA NELLA FILOSOFIA POSTMODERNA DI HUGO TRISTRAM ENGELHARDT}

Per comprendere come la bioetica postmoderna si ponga dinanzi al tema della fecondazione assistita, è sufficiente presentare la posizione di un esponente autorevole del variegato mondo della bioetica postmoderna americana: Hugo Tristram 
Engelhardt ${ }^{1}$. Il suo pensiero, altro non è che il pensiero di alcuni filosofi italiani e polacchi dei giorni nostri ${ }^{2}$ che tanto hanno attinto dalla letteratura anglosassone. Engelhardt scrive dal punto di vista di una società secolarizzata, anche quando si dice cristiana e cattolica.

\subsection{Concezione empiristica della persona}

Engelhardt definisce la persona in senso stretto come agente morale, dunque, l'individuo umano adulto che fa parte della comunità morale in quanto dotato di autonomia (capacità di autocoscienza e di autodeterminazione), di razionalità (capacità attuale di esercizio di raziocinio) e di attribuzione di un senso morale minimo (capacità di fare promesse e di avanzare pretese morali) ${ }^{3}$.

Da tali premesse segue la non identificazione tra vita umana biologica e vita umana personale ${ }^{4}$. Queste due nozioni sono rimaste legate l'una all'altra fino a quando la tradizione filosofica occidentale ha ritenuto che il corpo umano fosse guidato da un'anima razionale, considerata come la fonte dell'agire morale ${ }^{5}$. Questa concezione è entrata in crisi con lo sviluppo delle scienze della vita, "che ci hanno aiutato a comprendere come le funzioni biologiche si formino senza necessariamente fare appello ad un principio vitale, chiamato anima. La vita, intesa

${ }^{1}$ Hugo Tristram Engelhardt, Jr., filosofo americano di orgine tedesca, nato nel 1941, si è laureato in filosofia presso l'Università del Texas nel 1969 e in medicina presso l'Università di Tulane nel 1972. Negli anni 1969-1970 ha studiato all'Università di Bonn in Germania. È diventato professore di filosofia e di bioetica presso il dipartimento di Medicina del Baylor College of Medicine a Houston, Texas, USA, e presso il dipartimento di Filosofia della Rice University. Ė membro del Center for Medical Ethics and Health Policy di Baylor, e responsabile di numerose testate mediche e filosofiche. È autore di oltre 250 articoli e capitoli di libri, è inoltre co-editore di oltre 25 volumi. Tra le sue pubblicazioni vanno ricordate: due edizioni di The Foundations of Bioethics (1986, 1996), (Manuale di bioetica, 1991, 1999), Bioeethics and Secular Humanism: The Search for a Common Morality (1991) e The Journal of Medicine and Philosophy. E' inoltre co-editore della rivista Christian Bioethics e della collana Clinical Medical Ethics. Le sue opere sono state tradotte in diverse lingue.

${ }^{2}$ Cf. M. Mori, "La fecondazione artificiale", Laterza, Bari 1995; S. Veca, "Etica e verità: saggi brevi", Casagrande 2009.

${ }^{3}$ Cf. H.T. Engelhardt, Manuale di bioetica, Feltrinelli, Milano 1991, 156.

${ }^{4}$ Con la vita umana personale capisce che questa vita è tipica degli agenti morali, i quali, in forza della loro intelligenza, libertà e autocoscienza, possono disporre di sé e compiere atti qualificabili moralmente.

${ }^{5}$ Cf. M. Cascone, "Persona in prospettiva bioetica", Rivista di teologia morale 139 (2003), 401-416, 404. 
come processo biologico, ha cessato di essere un mistero dal momento che la scienza è in grado di spiegarla compiutamente e di riprodurla".

Applicando questo principio allo sviluppo iniziale della vita umana, Engelhardt afferma che: "Non tutti gli uomini sono persone. Non tutti gli uomini sono autocoscienti, razionali e capaci di concepire la possibilità del biasimo e della lode. Feti, infanti, ritardati mentali gravi e malati o feriti in coma irreversibile sono umani, ma non sono persone. Sono membri della specie umana, ma di per sé non hanno lo status di membri della comunità morale laica. Non possono né biasimare né essere biasimati, né lodare né essere lodati; non sono in grado nemmeno di fare promesse, di concludere contratti o di accordarsi su un atto di beneficenza. Non sono attori primari dell'impresa morale laica. Solo le persone hanno questo status"7.

\section{L'essere umano è persona e, quindi soggetto morale e soggetto di diritti, solo quando manifesta quelle caratteristiche peculiari (autocoscienza, razionalità, senso morale e libertà $)^{8}$, che lo rendono capace di entrare in un rapporto contrattuale sociale e comunitario, e non in quanto appartenente alla specie Homo sapiens ${ }^{9}$.}

Ne consegue che: "non tutti gli esseri umani sono uguali"10, oltre che l'esclusione, o meglio, il graduale riconoscimento dello statuto morale e giuridico secondo una scala progressiva numerata degli esseri umani potenziali e probabili persone ${ }^{11}$.

${ }^{6}$ Ibid., 404.

${ }^{7}$ Cf. H.T. Engelhardt, Manuale di bioetica..., 159.

${ }^{8}$ Ibid., 160.

${ }^{9}$ Ibid.

${ }^{10}$ Ibid., 155.

${ }^{11}$ Precisa Engelhardt: "Dal punto di vista della morale laica generale e della sua bioetica, si possono fare le seguenti distinzioni preliminari. C'è un senso del termine "persona" per il quale esso è sinonimo di agente morale e che io ho indicato anche come persona in senso 
Queste posizioni teoriche di approccio differenziato verso le persone possono condurre a delle conseguenze pratiche che Engelhardt colloca nel contesto della morale laica generale, quando scrive: "la sollecitudine che abbiamo verso gli individui che non sono e non saranno mai persone in senso stretto, può essere inferiore a quella che abbiamo per le persone in senso stretto" ${ }^{\prime 2}$. Si può facilmente intuire in quale direzione tenda qui il ragionamento.

Nell'uomo, secondo Engelhardt, si devono distinguere il livello della vita biologica, quello della vita mentale e quello della vita della persona. Perché ci sia la persona umana, ci deve essere la vita mentale di livello superiore, mentre non basta che ci sia qualunque vita mentale. Non è plausibile sostenere che i feti siano persone in senso stretto. In effetti, non ci sono prove nemmeno per sostenere che gli infanti siano persone in tal senso. Con questo pensiero Engelhardt mette in discussione lo statuto morale non solo degli zigoti umani e degli embrioni ma anche degli infanti. Questo pensiero è condiviso da numerosi studiosi che si collocano in una dimensione riduzionista e rifiutano di riconoscere connotazioni personali alla vita umana prenatale, neonatale e postnatale, oltre che alla vita terminale, alla vita gravemente malformata e handicappata ${ }^{13}$.

stretto, ma che potremmo anche chiamare persona 1); alla persona in senso stretto si contrappone la persona in senso sociale, alle quale, come nel caso dei bambini piccoli, vengono accordati all'incirca gli stessi diritti della persona in senso stretto (potremmo chiamarla persona 2). Uno status di persona in senso sociale è anche quello che viene accordato a individui che non sono più persone, ma che lo sono state in passato e restano ancora capaci di qualche interazione minimale (persona 3 ), nonchè quello accordato a individui gravemente ritardati e dementi che non sono mai stati e non saranno mai persone in senso stretto (persona 4). Un altro possibile senso sociale di persona è quello che può attribuirsi a certi esseri umani gravemente menomati (per esempio, ai soggetti in coma grave e irrevisibile) incapaci di interagire perfino in ruoli sociali minimali (persona 5). La nozione apparentemente unica di essere umano come persona o come agente morale si frantuma in una molteplicità di sensi. (...) Le persone che sono agenti morale hanno diriti che fanno parte integrante della natura stessa della morale laica generale. I diritti delle persone in senso sociale sono una creazione di comunità particolari (...) (che possono prevedere anche istituzioni responsabili dell'assistenza sanitaria)"; Ibid., 172-173.

${ }^{12}$ Ibid., 378.

${ }^{13}$ Cf. P. Singer, Animal liberation, Random House, New York 1975; traduzione italiana: Liberazione animale, Il Saggiatore, Milano 2010; N. M. Ford, When did I begin? Conception of the human individual in history, philodophy and science, Cambridge University Press, Cambridge 1988; traduzione italiana: Quando comincio io?, Baldini \& Castoldi, Milano 1997. 
Tali affermazioni portano a considerare come il valore proprio di un feto non dipenda dal feto stesso, ma dalle persone alle quali esso appartiene, che ne determinano tale valore. Circa l'argomentazione sulla fecondazione artificiale egli spiega che "l'inizio della vita umana biologica non è immediatamente seguito dall'inizio della vita di una persona. Al contrario, nello sviluppo dell'uomo, trascorrono mesi di vita biologica prima che vi sia la prova evidente della vita di una mente e passano anni prima che vi sia la prova della vita di una persona"14.

Infatti, un feto può essere considerato possessore di un valore intrinseco se è il frutto del desiderio di una donna, mentre può essere persino odiato se non voluto o se generato handicappato o deforme. Anche nel caso della maternità surrogata, nella quale altre persone entrano in relazione con l'embrione, i diritti non sono diritti dell'embrione o del feto, ma di coloro che hanno stipulato il contratto. Sono coloro che hanno fatto o procreato lo zigote, l'embrione o il feto ad avere il diritto di determinare il suo valore in modo definitivo. Gli embrioni e i feti prodotti privatamente sono di proprietà privata ${ }^{15}$.

La distinzione sulla diversa dignità degli esseri umani, funzionale alla descrizione di persona, lo porta ad affermare che secondo una prospettiva laica non ha senso parlare di rispetto dell'autonomia per i feti, gli infanti e i ritardati mentali ${ }^{16}$. Pertanto tutti questi soggetti non vengono privati di nulla se trattati senza riguardo, dal momento che a causa del loro sviluppo incompleto o del loro handicap non posseggono dignità morale ${ }^{17}$. Engelhardt, è convinto che questo orientamento liberi la discussione da questioni metafisiche e limiti gli obblighi morali permettendo la non proliferazione degli individui improduttivi da sfamare ed assistere $^{18}$. È consapevole che questa suddivisione possa ingenerare controversie ${ }^{19}$, le quali peraltro possono essere superate se si comprende che la morale laica "non

${ }^{14}$ H.T. Engelhardt, Manuale di Bioetica..., 248.

${ }^{15}$ Cf. Ibid., 251.

${ }^{16}$ Ibid., 160.

${ }^{17}$ Ibid., Le persone con gravi handicap fisici o mentali porrebbero in essere obblighi morali troppo onerosi. Il valore di uno zigote, un embrione o un feto con una capacità di sentire di gran lunga inferiore a quella di un mammifero adulto, va valutato in rapporto al significato che gli conferiscono le persone effettivamente esistenti. Solo le tradizioni morali particolari, però, potranno valutare il corretto uso degli animali. La morale laica ambisce unicamente ad evitare che siano trattati in modo malevolo (cf. H.T. Engelhardt, Manuale di bioetica..., 164-168).

${ }^{18}$ Ibid., 164.

${ }^{19}$ Ibid., 161. 
è in grado di mettere in luce il valore canonico della vita biologica umana"20. Diverse sono le posizioni di chi si occupa della vita biologica. È tipico dei Comitati di Studio, sollecitati a dare soluzioni ai problemi mossi della prassi, lasciare alla filosofia il compito delle definizioni. Il personalismo ontologicamente fondato, invece, integra i dati biologici e quelli filosofici in un sistema coerente, nel quale la persona umana è considerata tale dal concepimento fino alla morte ${ }^{21}$. Il vitalismo in senso potenziale presenta notevoli diversificazioni. Si qualificano essenzialmente due raggruppamenti di pensiero. Per quanto riguarda l'embrione, ad esempio, il primo esprime una notevole diversità di opinioni. L'emergere certo dell'individuo è situato a diversi stadi dello sviluppo embrionale (dalle primissime ore del concepimento fino al quattordicesimo/quindicesimo giorno). Il secondo invece esige la comparsa dei primi segni di attività cerebrale e posticipa l'emergere dell'individuo ad un periodo che va dalla sesta alla ventesima settimana. Non mancano coloro che si spingono ancora più in là, ritenendo che si divenga persona solo dopo la nascita ${ }^{22}$.

Nella questione dell'aborto, seguendo le osservazioni di Armani, i criteri proposti da Engelhardt sono i seguenti:

- il feto non è persona;

- la donna ha il diritto di esercitare il controllo sul proprio corpo;

- la donna ha il diritto di prendere le proprie decisioni riguardo alla riproduzione $\mathrm{e}^{23}$.

Per comprendere la posizione di Engelhardt è necessario tener presente la sua concezione di persona, la sua antropologia, da cui logicamente deriva il rifiuto di riconoscere all'embrione - e non solo, ma anche al feto che come prodotto biologico non ha una vita mentale, che si svilupperà solo qualche tempo dopo la nascita, e al neonato, ecc. - lo statuto di persona ${ }^{24}$.

${ }^{20}$ Ibid., 164.

${ }^{21}$ Cf. M. Lomabardi Ricci, Fabbricare bambini? La questione dell'embrione tra nuova medicina e genetica, Vita e Pensiero, Milano 1996, 109-116.

${ }^{22}$ Cf. Ibid., 117-121.

${ }^{23}$ Cf. M. Aramini, Procreazione assistita..., 99-100.

${ }^{24}$ Cf. H.T. Engelhardt, Manuale di bioetica..., 163. Riflettendo sulle fasi iniziali della vita, ritiene, inoltre, poco convincente la nazione di potenzialità (cfr. idem., 163-166), fatta propria dalla teologia cattolica, secondo cui feti e bambini sono già da rispettare come persone e, per ciò stesso, sono portatori dei diritti attribuiti alle persone (cfr. Ibid., 163). 


\subsection{La disponibilità della natura umana}

L'atteggiamento di libertà della persona nei confronti degli esseri non personali mette in luce anche una determinata tipologia del rapporto tra persona e natura. Il contesto della riflessione di Engelhardt è quello di una concezione del rapporto uomo-natura, nel quale l'uomo utilizza tutti i mezzi disponibili per assoggettare la natura e controllarne gli effetti indesiderati. Nella sua antropologia Engelhardt ribadisce come l'uomo abbia tutti i diritti di manovrare la natura a seconda delle proprie esigenze e dei propri bisogni. La natura ha una rilevanza solo materialistica e meccanicistica, essa non ha nulla da dire all'uomo, non ha un fine, né un significato e, perciò, non può dare indicazioni e norme per il comportamento umano ${ }^{25}$. Engelhardt riconosce che la persona è limitata in qualche modo dalla sua natura, nella quale non c'è nulla di sacro ${ }^{26}$. Questo va compreso non nel senso che la natura umana contenga in sé una qualche forma di indicazione che vincoli la libertà personale nel suo intervento manipolatore; tutto ciò che si può tecnicamente fare è moralmente lecito nella visione morale laica rappresentata da Engelhardt. La comprensione del limite consiste, invece, nel fatto che, nonostante i desideri della persona siano infiniti, tuttavia essa non può tecnicamente servirsi della propria natura per realizzare ciò che vuole ${ }^{27}$.

L'assunto che la natura non ha nulla di sacro comporta che agli occhi della morale laica di Engelhardt il processo che guida la sua trasformazione ad opera della persona non può essere giustificato in base a nessun criterio orientativo basato su una verità oppure su un valore canonico e sostantivo. Ne consegue per Engelhardt che: "Non c'è ragione di presumere che dagli uomini debba derivare una sola specie. Potrebbero derivarne tante quante sono sia le possibilità attraenti di rimodellare sostanzialmente la natura umana per l'ambiente presente o per nuovi ambienti, sia le ragioni per rifiutarsi di prendervi parte" 28 .

\subsection{Critica dell'argomento di persona}

H. T. Engelhardt presenta quindi la visione funzonalistico-attualista del concetto di persona. Secondo questa posizione, l'embrione, il feto o il bambino malformato non sono persone, ma lo sono solo i figli nati e accettati dai genitori e, analogamente, gli adulti psichicamente handicappati o quelli che hanno perso la coscienza,

\footnotetext{
${ }^{25}$ Cf. H.T. Engelhardt, Manuale di Bioetica..., 248.

${ }^{26}$ Ibid., 430.

${ }^{27}$ Ibid., 434-435.

${ }^{28}$ Ibid., 436.
} 
ad esempio in stato vegetativo persistente o in fase terminale, non sono persone ${ }^{29}$. Secondo Sgreccia la posizione funzionalistico-attualista non offre una definizione metafisica della persona. Questa posizione conduce ad una riduzione del concetto di persona alle sole funzioni che è in grado di svolgere. Così si arriva alla definizione della persona solo in base a ciò che appare e non ciò che è. Ma, contemporaneamente, ciò "significa tradire lo stesso significato dell'essenza uomo. Questo perché, una volta abolita la differenza tra sostanza/esistenza e le sue funzioni/operazioni, il soggetto è interpretato solo come una funzione che si esprime in una somma di atti, non come atto primo di essere di una sostanza che costituisca il luogo d'incontro di tutti gli altri atti, rendendoli possibili e sostenendoli"30.

F. Campagnoni rifiuta la distinzione tra individuo umano e persona, perché dal punto di vista ontologico ambedue i concetti significano la stessa natura ed esprimono la medesima sostanza. Anche se è vero che la stessa persona durante la sua vita attraverso diverse tappe biologiche e fisiologiche, nonostante tutto, queste tappe e i cambiamenti intrinsecamente collegati con esse non cambiano la sostanza della persona. Campagnoni rifiuta la negazione della differenza ontologica tra l'uomo e gli altri esseri non-umani utilizzando l'argomentazione filosofica di E. Agazzi, per il fatto che "un essere può diventare soltanto ciò che è già per sua natura. Questo principio assolutamente generale dev'essere applicato anche alla persona: nulla può diventare una persona senza già essere una persona" ${ }^{31}$. In altre parole, "ciò che vive è, ed è come è e non può essere altrimenti"32.

Una posizione più radicale e critica verso la definizione della persona secondo Engelhardt si trova in L. Ciccone. Egli (e altri ${ }^{33}$ ) mette in evidenza la negazione

${ }^{29}$ Cf. E. Sgreccia - M.L. Di Pietro, La persona e il modello..., 151-152; e anche E. Sgreccia, La persona e la vita..., 38-41.

${ }^{30}$ Ibid., 155;

${ }^{31}$ E. Agazzi, “L'essere umano come persona”, Perfil 25 (1992), 28-39, 37.

${ }^{32} \mathrm{~F}$. Compagnoni, "Chi è persona umana? Contributo teologico al dibattito bioetico sull'inizio e la fine della vita dell'uomo", in La bioetica alla ricerca della persona negli stati di confine, ed. C. Viafora, Padova 1994, 97-114, 110.

${ }^{33}$ Una critica della posizione di H.T. Engelhardt riguardante la categoria di persona è stata sviluppata pure da L. Alici: "Dinanzi a questa posizione di Engelhardt si potrebbe anzitutto osservare che qui siamo in presenza di un possibile uso ideologico del termine persona, nella misura in cui si tende ad occultare la sua valenza ontologica ed etica, adoperandolo piuttosto come sinonimo di individuo umano soggetto di bisogni. (...) In questo modo l'uso ideologico di persona viene alla luce in maniera ancora più esplicita: ci si richiama alla persona non per innalzare il dibattito della bioetica a livello filosofico, bensi per evitare tale dibattito e ridursi 
della differenza ontologica tra uomo ed esseri non umani da parte di Engelhardt: "L'enorme distanza tra uomo e animale (...) è un dato di fatto evidente, che si impone a chiunque non si lasci bendare gli occhi della ragione da pregiudizi e ideologie. (...) Riconoscere all'essere umano una superiorità qualitativa enorme e singolare non è un'opinione, una teoria, ma un dato di fatto" ${ }^{34}$.

In sintesi, Engelhardt nega l'equivalenza ontologica tra individuo della specie umana e persona. La persona nascerebbe solo dal riconoscimento, dal permesso, dal contratto degli altri.
La dignità della persona si intende come una qualità permessa solo a certi individui, ma sicuramente non a tutti, da parte degli individui più forti che decidono sui concetti dominanti della società. Si vede in questo modo che la persona è privata del suo fondamento ontologico e che il contenuto del concetto di persona diventa oggetto di una convenzione o di un contratto ${ }^{35}$.

In tale maniera si è passato dal pensiero forte o ontologico a quello debole o postmoderno.

ad una casistica, ad un compromesso pratico tra posizioni diverse", (L. Alici, "Temi di bioetica nella filososfia morale", in La Bioetica. Profili culturali, sociali, politici e giuridici, ed. G. Dalla Torre - L. Palazzani, Roma 1997, 121-144, 127-128.

Per l'analisi e la critica del contrattualismo sociale di H.T. Engelhardt a riguardo della riabilitazione delle persone gravemente disabili che non sono considerate come persone, vedi: E. Sgreccia - A. Fiore - A.G. Spagnolo, "Bioetica, disabilità e riabilitazione", in Bioetica. Manuale per i Diplomi Universitari della Sanità, ed. E. Sgreccia - A.G. Spagnolo - M.L. Di Pietro, Milano 1999, 509-531, 513-514.

${ }^{34}$ L. Ciccone, "Una critica a "Ripensare la vita” di Peter Singer", Bioetica. Rivista interdisciplinare 1 (1997), 63-75, 74.

${ }^{35}$ Cf. G. Cottier, "Morale e diritto in bioetica: Il paradigma dei diritti umani", in Quando morire? Bioetica e diritto nel dibattito sull'eutanasia, ed. C. Viafora, Padova 1996, 87-116, 95-98. 


\subsection{La fecondazione artificiale}

Alla luce di queste considerazioni e su tali basi, ecco il nucleo essenziale della posizione postmoderna di Engelhardt nei confronti delle tecniche riproduttive: "Diventa molto difficile porre delle restrizioni alla fecondazione in vitro fatta con lo scopo di generare un bambino sano per genitori che non sarebbero altrimenti in grado di riprodursi. (...) L'uso della tecnologia per creare dei bambini è intrinseco al fine di rendere il mondo congeniale alle persone. Tali interventi possono essere considerati scorretti in via di principio solo facendo appello a speciali premesse teologiche o ideologiche"36.

Chiaramente Engelhardt dinanzi alla fecondazione artificiale si pone con atteggiamento favorevole e condanna le obiezioni che si frappongono sull'utilizzo di tale pratica. L'obiezione sull'innaturalità della masturbazione per il prelievo del seme maschile viene fortemente contestata poiché essa, oltre ad essere ritenuta naturale, è anche il frutto dell'evoluzione e dell'uso delle capacità della specie umana rispetto a quella animale. Infine non sarà possibile condannare la fecondazione in vitro perché questa puó comportare lo spreco di embrioni fecondati. Non si arreca danno ad alcuna persona eliminando embrioni in eccesso, prodotti nel processo di fecondazione in vitro ${ }^{37}$.

Secondo Engelhardt, la FIVET, pianificata in modo razionale, è naturale per gli esseri razionali. L'uso della tecnologia per creare dei bambini è un servizio per rendere il mondo più vivibile. "La fecondazione in vitro e le tecniche che ci permettono di studiare e controllare la riproduzione umana sono strumenti moralmente neutri per la realizzazione di fini umani di grandissima importanza, legati alla realizzazione del bene altrui, dei figli per i genitori sterili e di una salute migliore per i figli che nasceranno"38.

L'eliminazione degli embrioni, secondo Engelhardt, non ha alcun valore. Egli, pur con queste affermazioni estreme, nella liceità di molte situazioni e dei suoi pensieri, dichiara di essere una persona di fede ${ }^{39}$. Per questi motivi, nel valutare la filosofia postmoderna di Engelhardt, si può dire che egli confonda il piano

${ }^{36}$ H.T. Engehardt, Manuale di bioetica..., 275.

${ }^{37}$ Cf. Ibid., 275-277.

${ }^{38}$ Ibid., 278.

${ }^{39}$ L'affermazione: "Io sono un credente cattolico", si trova al termine di un'intervista pubblicata con il titolo "La bioetica nell'era postmoderna", Notizie di Politeia, 24 (1994), cit. in F. Compagnoni (a cura di), Etica della vita, San Paolo, Milano 1996, 20. 
ontologico dell'essere con quello accidentale dell'agire, arrivando ad affermare che il riconoscimento della persona non sarebbe dipendente dalla presenza di natura razionale, ma da fattori esterni. Pertanto un individuo, incapace di manifestare tutte le sue capacità razionali, potrebbe non ottenere, o ottenere solo in parte, il riconoscimento del suo stato di persona. Nella sua visione postmoderna, Engelhardt giustifica la sottovalutazione di tutti coloro che non fanno ancora parte della comunità (embrioni, feti e bambini), i cui diritti dipenderebbero pertanto dagli adulti e, in definitiva, non sono considerati come persone. Per spiegare meglio il valore che in genere viene attribuito al feto e al bambino già nato, Engelhardt sostiene che bisogna fare riferimento non già al feto stesso o al bambino, ma al significato che questa vita biologicamente umana assume per la donna che l'ha concepita e per le altre persone direttamente interessate ad essa ${ }^{40}$. Questo vuol dire che il feto non ha valore in se stesso, ma possiede solo il valore che gli altri, a cominciare dalla madre, gli attribuiscono. Genitori, nonni, zii possono tributare al feto e all'infante un grande valore, ma possono anche assegnargli un valore negativo, quando per esempio il feto è deformato e le circostanze del concepimento sono state violente o quando nasce un bambino gravemente handicappato sul piano psichico. La conseguenza di questo ragionamento è ovvia: "almeno nell'ottica della morale laica generale coloro che hanno prodotto un feto hanno per primi il diritto di determinarne efficacemente l'uso" ${ }^{41}$.

Il rapporto con queste non persone può essere stabilito solo su criteri utilitaristici, ossia tenendo conto di ciò che è più vantaggioso per loro stessi e per il maggior numero di persone interessate. Per essere più chiaro, Engelhardt sostiene che la norma generale di proteggere i neonati e di averne cura "ammette eccezioni in presenza di una bassa qualità dei risultati e dei costi elevati del trattamento"42. Così egli spiega a tal proposito: "Qualcuno potrebbe desiderare di evitare che vengano al mondo persone in senso stretto con handicap gravi, proprio in considerazione del fatto che potrebbero avanzare pretese molto onerose in termini di doveri di beneficenza"43. Assodato il fatto che bisogna mettere al mondo solo persone che possano godere di una buona qualità della vita, "il dovere di

\footnotetext{
${ }^{40}$ Cf. H.T. Engelhardt, Manuale di bioetica..., 276.

${ }^{41}$ Ibid.

${ }^{42}$ Ibid., 287.

${ }^{43}$ Ibid., 288.
} 
preservare la vita di un neonato generalmente viene meno con il diminuire della possibilità di successo, nonché della qualità e della quantità della sua vita, oltre che con l'aumentare dei costi del conseguimento di tale qualità" ${ }^{44}$. La conclusione a questo punto è ovvia: "In termini morali laici generali non c'è ragione per sostenere che sia moralmente sbagliato volere intenzionalmente la morte degli infanti che non si è tenuti a mantenere in vita con un trattamento appropriato" ${ }^{45}$.

Engelhardt applica tutti i canoni dell'utilitarismo, facendo leva sull'onerosità di una vita senza prospettive, che andrebbe soppressa per pietà o compassione, specialmente per evitare sofferenze a quelli che sono in grado di soffrire: i genitori, gli adulti, la comunità delle persone in senso stretto, ossia tutti coloro che, in un'ottica contrattualistica, sono chiamati a decidere non solo per il vantaggio collettivo, ma anche a nome di coloro che, non potendosi qualificare persone in senso stretto, non sono in grado di esprimere un proprio permesso o consenso. Su questa base e con questo spirito Engelhardt giustifica la liceità delle tecniche di riproduzione umana.

Riassumendo si nota che questo approccio alla bioetica non è ontologicamente fondato, non riconosce nessuna gerarchia ontologica di valori. Il valore della vita umana è relativo, senza riconoscimento della gradualità e delle differenze ontologiche ed assiologiche. Lo statuto della persona umana viene riconosciuto solo agli individuali autocoscienti e che possono esprimere la loro volontà. Il concetto di qualità della vita non è fondato né dal punto di vista logico, né ontologico, né etico. Perché l'uomo e la sua vita non hanno nessun senso ontologico. L'uomo e la sua vita valgono solo in tanto in quanto sono strumenti o oggetti di soddisfazione di bisogni personali e comunitari.

\section{BIBLIOGRAFIA}

Agazzi E., "L'essere umano come persona”, Perfil 25 (1992), 28-39.

Alici L., "Temi di bioetica nella filososfia morale", in La Bioetica. Profili culturali, sociali, politici e giuridici, ed. G. Dalla Torre - L. Palazzani, Roma 1997, 121-144.

Aramini M., La procreazione assistita. Scoprire il senso di un nuovo modo di nascere, San Paolo, Milano 1999.

${ }^{44}$ Ibid., 289.

${ }^{45}$ Ibid., 293. 
Cascone M., "Persona in prospettiva bioetica”, Rivista di teologia morale 139 (2003), 401-416.

Ciccone L., "Una critica a "Ripensare la vita" di Peter Singer", Bioetica. Rivista interdisciplinare 1 (1997), 63-75.

Compagnoni F., (a cura di), Etica della vita, San Paolo, Milano 1996.

Compagnoni F., "Chi è persona umana? Contributo teologico al dibattito bioetico sull'inizio e la fine della vita dell'uomo", in La bioetica alla ricerca della persona negli stati di confine, ed. C. Viafora, Padova 1994, 97-114.

Cottier G., "Morale e diritto in bioetica: Il paradigma dei diritti umani", in Quando morire? Bioetica e diritto nel dibattito sull'eutanasia, ed. C. Viafora, Padova 1996, 87-116.

Engelhardt H.T., Manuale di bioetica, Feltrinelli, Milano 1991.

Ford N.M., When did I begin? Conception of the human individual in history, philodophy and science, Cambridge University Press, Cambridge 1988; traduzione italiana: Quando comincio io?, Baldini \& Castoldi, Milano 1997.

Lomabardi Ricci M., Fabbricare bambini? La questione dell'embrione tra nuova medicina e genetica, Vita e Pensiero, Milano 1996.

Mori M, "La fecondazione artificiale”, Laterza, Bari 1995.

Sgreccia E. - Di Pietro M.L., La persona e il modello personalista, Bioetica. Manuale per i Diplomi Universitari della Sanità, ed. E. Sgreccia - A.G. Spagnolo - M.L. Di Pietro, Milano 1999, 155-162.

Sgreccia E. - Fiore A. - Spagnolo A.G., "Bioetica, disabilità e riabilitazione”, in Bioetica. Manuale per i Diplomi Universitari della Sanità, ed. E. Sgreccia - A.G. Spagnolo - M.L. Di Pietro, Milano 1999, 509-531.

Singer P., Animal liberation, Random House, New York 1975; traduzione italiana: Liberazione animale, Il Saggiatore, Milano 2010.

Veca S., "Etica e verità: saggi brevi", Casagrande 2009.

\section{Biogram}

Jacek Rosa (Siedlce) - ks. dr, prezbiter diecezji siedleckiej, bioetyk, prowadzi badania nad zagadnieniami bioetycznymi w perspektywie personalizmu chrześcijańskiego i zagadnień bezpieczeństwa osoby ludzkiej, adiunkt w Wyższej Szkole Policji w Szczytnie. Adres e-mail: jacekrosa79@gmail.com 